\title{
Disordered photonics behavior from terahertz to ultraviolet of a three-dimensional graphene network
}

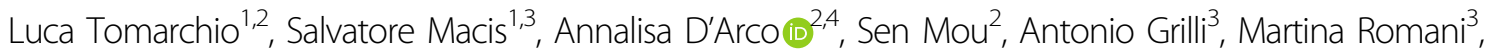 \\ Mariangela Cestelli Guidi ${ }^{3}$, Kailong $\mathrm{Hu}^{5}$, Suresh Kukunuri ${ }^{6}$, Samuel Jeong $\mathbb{1}^{6}$, Augusto Marcelli ${ }^{3}$, Yoshikazu Ito ${ }^{6}$ and \\ Stefano Lupi (i) ${ }^{1,3}$
}

\begin{abstract}
The diffusion of light by random materials is a general phenomenon that appears in many different systems, spanning from colloidal suspension in liquid crystals to disordered metal sponges and paper composed of random fibers. Random scattering is also a key element behind mimicry of several animals, such as white beetles and chameleons. Here, random scattering is related to micro and nanosized spatial structures affecting a broad electromagnetic region. In this work, we have investigated how random scattering modulates the optical properties, from terahertz to ultraviolet light, of a novel functional material, i.e., a three-dimensional graphene (3D Graphene) network based on interconnected high-quality two-dimensional graphene layers. Here, random scattering generates a high-frequency pass-filter behavior. The optical properties of these graphene structures bridge the nanoworld into the macroscopic world, paving the way for their use in novel optoelectronic devices.
\end{abstract}

\section{Introduction}

Due to the advances of nanotechnology and the discovery of various $2 \mathrm{D}$ functional materials, in recent years there has been a growing interest for extending their extraordinary properties to three-dimensional (3D) ordered and disordered structures.

This is particularly true for graphene, since its unique

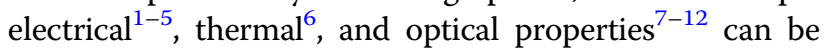
extended into 3D structures by stacking high-quality monolayer graphene sheets into a wide variety of three-dimensional networks ${ }^{13-15}$. These 3D structures show performances beyond 2D graphene devices, ranging from supercapacitors ${ }^{16-28}$, lithium batteries ${ }^{29-40}$ and electrocatalysts $^{41-51}$, to photodetectors ${ }^{52-54}$, biochemical

\footnotetext{
Correspondence: Stefano Lupi (stefano.lupi@roma1.infn.it)

'Department of Physics, Sapienza University, Piazzale Aldo Moro 5, 00185 Rome, Italy

${ }^{2}$ INFN section of Rome, P.Le Aldo Moro, 2, 00185 Rome, Italy

Full list of author information is available at the end of the article
}

applications ${ }^{55-57}$, and transistors ${ }^{58,59}$. Dirac plasmonic behaviors have also been observed in 3D nanoporous graphene. Plasmon absorption can be modulated by changing both the chemical doping and the pore size ${ }^{60}$. Moreover, through the photo/thermoacoustic effect, 3D Graphene sponges provide an efficient transduction of light into sound, covering a very broad acoustic emission range from infrasound (few $\mathrm{Hz}$ ) to deep ultrasound (few $\mathrm{MHz})^{61-63}$. Despite those broad applications, very little information is known about the light interaction with 3D graphene structures, where the network composed of pores and branches is expected to interact collectively with a broadband portion of the electromagnetic spectrum ${ }^{64}$.

In this work, we measure the optical properties of undoped and nitrogen-doped 3D porous graphene networks over a broadband spectral range, going from Terahertz (THz) to ultraviolet (UV), demonstrating optical high-pass filtering of these structures mainly determined by random scattering effects. The transmission of light 
across a random network, composed of branches and pores, depends both on the 3D morphology of the system and on the optical properties of the supporting material. Variability in the pore size induces changes in the system connectivity, and thus in the propagation and scattering of light at various wavelengths. Through the study of the broadband transmission of graphene networks, we emphasize their emergent complex optical properties which can be extended to other $2 \mathrm{D}$ functional materials.

\section{Results and discussion}

In this paper, we measured two nanoporous graphene samples composed of high-quality single layer graphene ${ }^{65}$, both with an average pore size of $\approx 100( \pm 50) \mu \mathrm{m}$. A first sample (S1) is undoped, while the second (S2) has been doped with nitrogen and shows a DC conductivity of approximately $70 \mathrm{~S} / \mathrm{cm}$, nearly a factor 140 larger than the undoped conductivity $(0.5 \mathrm{~S} / \mathrm{cm})$. Both samples have a thickness of approximately $1 \mathrm{~mm}$ and are composed on average of 12 graphene layers. Their 3D structures were characterized by scanning electron microscopy (SEM) and optical microscopy. A third sample (S3) with an average pore size of $\approx 80( \pm 50) \mu \mathrm{m}$ is presented in the Supplementary Information $(\mathrm{SI})^{65}$. Their 3D structures have been characterized by scanning electron microscopy (SEM) and optical microscopy. A macroscopic optical image of the undoped sample S1 is shown in Fig. 1a, while a SEM image is shown in Fig. 1b. The optical image of a branch is finally shown in Fig. 1c, where a roughness of hundreds of nanometers related to interconnected graphene layers can be observed. Similar structures are found for doped sample S2 (see Fig. S1, $\mathrm{S} 2, \mathrm{~S} 3$, for a comparison) ${ }^{65}$. The broadband transmittance curves from terahertz (THz) to ultraviolet (UV) measured for the S1 and S2 samples are shown in Fig. 2 and in Fig. S4 for the S3 sample ${ }^{65}$. In the inset of Fig. 2 the transmittance is shown as a function of the wavelength. They show a highfrequency pass-filter behavior with a rapid increase in the transmittance from $\mathrm{THz}$ to mid-IR (MIR). Here, the transmittance reaches a saturation value corresponding to nearly 13\% for both S1 and S2 samples. Below $1 \mathrm{THz}$, both curves converge to zero. The undoped S1 and doped S2 samples show a similar optical behavior, regardless of the layer conductivity, which varies from $0.5 \mathrm{~S} / \mathrm{cm}(\mathrm{S} 1)$ to $70 \mathrm{~S} / \mathrm{cm}(\mathrm{S} 2)$.

Given the equivalent 3D structures of S1 and S2, the broadband transmittance behavior (Fig. 2) suggests that their nano and microspatial morphology dominates the light-matter interaction through scattering processes, rather than their intrinsic absorption. This demonstrates, in our opinion, that the optical properties of 3D graphene samples are dominated by scattering, rather than by the intrinsic absorption in the graphene layers.

This is also confirmed by the absence of absorptive features in the UV spectral range, where graphene is expected to host interband electronic transitions ${ }^{66,67}$. An explanation for the absence of UV absorption might come from the branch surface, as shown in Fig. 1c, where a surface roughness on a sub-wavelength scale suggests a pronounced scattering contribution, as already highlighted in similar works $^{64}$. Moreover, in the integrated infrared transmittance between 2000 and $3000 \mathrm{~cm}^{-1}$ of a single polygon (Fig. $1 \mathrm{~d}$ for the $\mathrm{S} 1$ sample), the integrated transmittance is nearly $1 \%$ in the branch regions, reaching values between 10 and $20 \%$ in the empty regions. A similar image is shown in $\mathrm{SI}^{65}$ for the S2 doped sample. The similarity between S1 and S2 further confirms that doping has a marginal role in the optical properties of the 3D graphene network.

To understand the role of scattering in the optical transmittance behavior, we developed goniophotometric measurements (see "Methods" and Fig. S5 in $\mathrm{SI}^{65}$ for a technical discussion) for two different wavelengths: $780 \mathrm{~nm}\left(\sim 13,000 \mathrm{~cm}^{-1}\right)$, falling in the broadband transmittance plateau, and at $7 \mu \mathrm{m}\left(\sim 1400 \mathrm{~cm}^{-1}\right)$, on the transmittance rising edge (see Fig. 2). Here, one measures the scattered light intensity in the far-field at a fixed distance $(30 \mathrm{~cm})$ with respect to the sample position (see Fig. 3a), scanning different horizontal $(\theta)$ and vertical $(\Phi)$ angular positions. As scattering shows a cylindrical symmetry $(\theta=\Phi)$, in the following we will discuss only the horizontal $\theta$ dependence. A $0.5 \mathrm{~mm}$ pinhole located in front of the detector provides a $0.1^{\circ}$ sampling of $\theta$.

Figure $3 \mathrm{~b}$ shows the $\theta$ dependent scattered intensities for the incident and transmitted beams of S1 sample, normalized to the zero angle incident signal. Similar results were measured for the S2 samples. Incident intensities at $7 \mu \mathrm{m}$ (light blue diamonds) and $780 \mathrm{~nm}$ (blue stars) can be described by the same Gaussian function and its angle distribution reflects the intrinsic laser beam divergence. The transmitted intensities are instead distributed at larger angles, suggesting a valuable contribution from scattering. Indeed, above nearly $1.5^{\circ}$ angle, the $7 \mu \mathrm{m}$ transmitted intensity (yellow diamonds) reaches a higher plateau of approximately one order of magnitude larger than the $780 \mathrm{~nm}$ signal (red stars). This suggests an increasing role of scattering with decreasing frequency.

To link the transmittance values at $780 \mathrm{~nm}$ and $7 \mu \mathrm{m}$ as shown in Fig. 2 to the goniophotometric data (Fig. 3b), we need to integrate the latter over the horizontal and vertical scattering angles. Given the scattering cylindrical symmetry $(\theta=\Phi)$, the integrated transmittance $T(\theta, \lambda)$, up to an angle $\theta$ and as a function of wavelength $\lambda$, will take the form

$$
T(\theta, \lambda)=\left[\int_{S(\Theta)} I_{T}(s, \lambda) \mathrm{d} s\right] /\left[\int_{S(\Theta)} I_{0}(s, \lambda) \mathrm{d} s\right]
$$

where $I_{T}$ and $I_{0}$ are, respectively, the transmitted and incident intensities as a function of wavelength and at a 

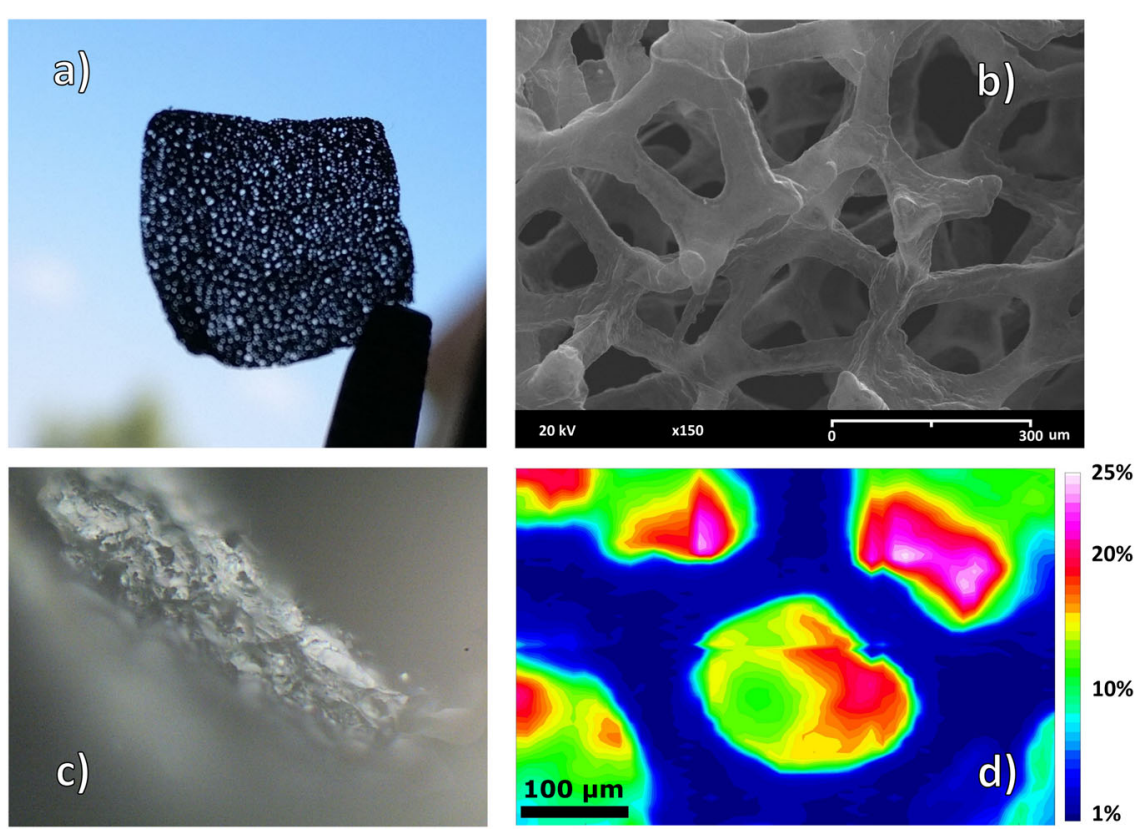

Fig. 1 Magnification of the 3D graphene network morphology. a Visible image of the graphene 3D network (S1 sample). b SEM $\times 150$ magnified image of the 3D graphene S1 sample. The 3D network is composed by several polygonal structures connected to each other. c Image of a S1 single branch obtained through an optical JASCO NR5100 microscope. The picture has been reconstructed through a focus stacking process applied to 20 raw pictures and shows an intrinsic roughness of hundreds of nanometers related to interconnected graphene layers. $\mathbf{d}$ Integrated infrared transmittance between 2000 and $3000 \mathrm{~cm}^{-1}$ of a single polygon of the undoped S1 sample, as taken by a $64 \times 64$ pixel Focal Plane Array (FPA) MCT detector coupled to a Vertex $70 \mathrm{~V}$ Bruker interferometer.

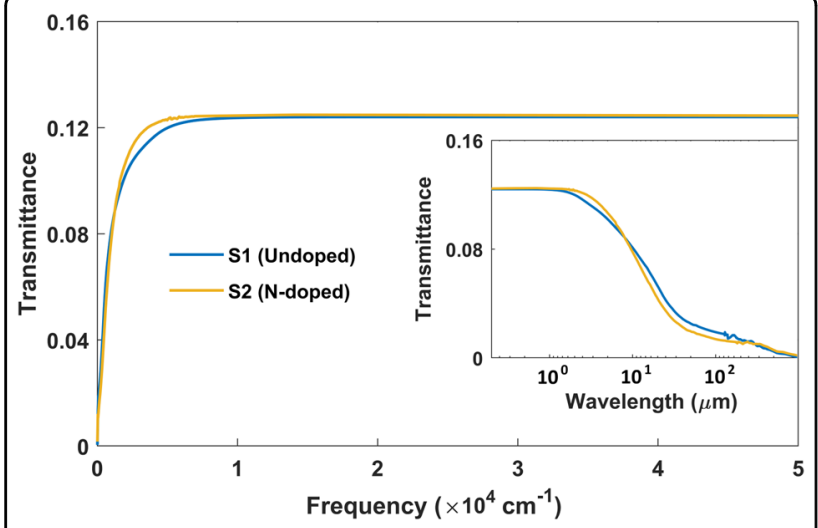

Fig. 2 Optical transmittance $T(\omega)$ of S1 and S2 3D graphene samples from THz to UV. T( $\omega)$ shows a high-pass-filter behavior, with a rapid increase from THz to MIR (nearly $5000 \mathrm{~cm}^{-1}$ ), and saturating to a constant value in the VIS and UV range (about 0.13 for both samples). The N-doped S2 sample does not show remarkable differences with respect to the undoped S1 one with the same average morphology. Transmittance data are shown in the inset as a function of wavelength.

specific surface section $s$ (identified by the same angular deviation), and the integral spans over the surface $S(\Theta)$ scanned by the detector (Fig. 3a). The values for $T(\theta, \lambda)$ are shown in Fig. 3c, where the integrated transmittance values for the $780 \mathrm{~nm}$ and $7 \mu \mathrm{m}$ wavelengths converge to the corresponding results of Fig. 2 at approximately $5^{\circ}$. This angle is in good agreement with the collection angle of the Bruker Vertex interferometer $\left(\sim 5^{\circ}\right)$ used for acquiring the transmittance data of Fig. 2. From the data of Fig. 3b, one can extract the fraction of scattered light $I_{s}$ vs. $\theta$ as ${ }^{65}$

$$
I_{s}(\theta)=I_{T}(\theta)-I_{0}(\theta) T(\theta=0)
$$

where

$$
T(\theta=0)=\frac{I_{T}(\theta=0)}{I_{0}(\theta=0)}
$$

is the transmittance at normal incidence used as a reference value for the unscattered light across the beam waist. The result for the scattered intensity is shown in Fig. 3d, after being normalized by the total transmitted intensity $I_{T}$ at any angle. This figure suggests a rapid increase in the scattering contribution to the angle-resolved transmittance, reaching its peak value (100\% of scattered light) at nearly $1^{\circ}$ angle. The slightly more rapid increase at $7 \mu \mathrm{m}$, with respect to $780 \mathrm{~nm}$, suggests a stronger scattering effect at higher wavelengths, particularly toward the $\mathrm{THz}$ range.

Goniophotometric results highlight how scattering plays the major role in setting the transmittance value at both $780 \mathrm{~nm}$ and $7 \mu \mathrm{m}$. The nearly one order of 

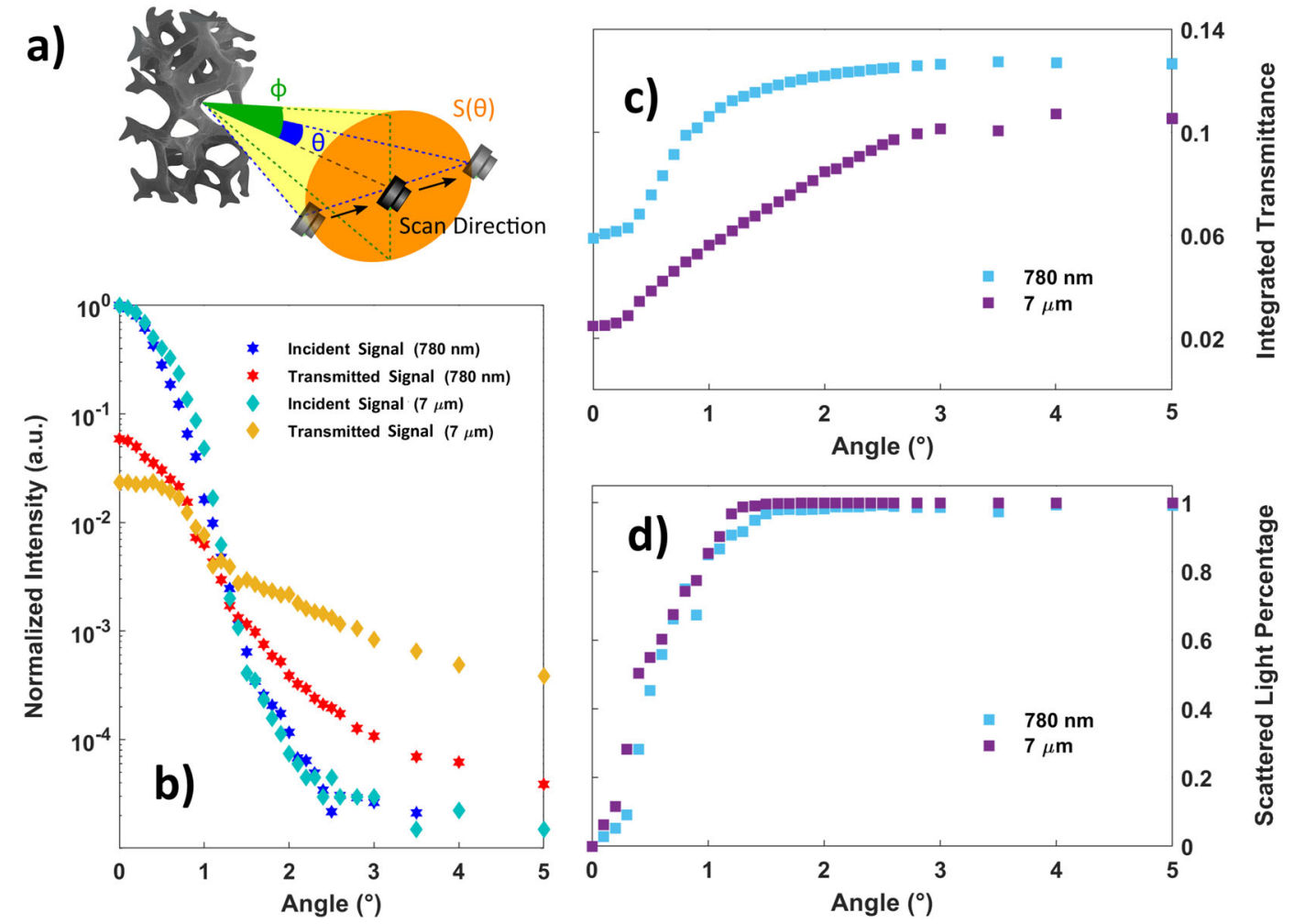

Fig. 3 Goniphotometric mesurements at $\mathbf{7 8 0} \mathrm{nm}$ and $7 \mu \mathrm{m}$. a Detection geometry of the goniophotometric data. $\theta$ is the horizontal scattering angle and $\Phi$ the vertical one. Due to the cylindrical symmetry $(\theta=\Phi)$, only the horizontal angle $(\theta)$ has been scanned with a sensitivity of $0.1^{\circ}$. b Incident and transmitted intensities of S1 sample at different $\theta$ angles for the $780 \mathrm{~nm}$ (blue and red stars) and $7 \mu \mathrm{m}$ (light blue and yellow diamonds) wavelengths. $\mathbf{c}$ Integrated transmittance $T(\theta, \lambda)$ for the S1 sample as a function of the scattering angle $\theta$, as computed from Eq. 1 for $780 \mathrm{~nm}$ (light blue squares) and $7 \mu \mathrm{m}$ (violet squares) wavelengths, respectively. $T(\theta, \lambda)$ values converge rapidly to the transmittance as measured in Fig. 2, supporting the idea that scattering is the key process in the light-3D Graphene interaction. $\mathbf{d}$ Fraction of scattered light for the S1 sample for both $780 \mathrm{~nm}$ (light blue squares) and $7 \mu \mathrm{m}$ (violet squares) wavelengths, as computed from Eq. 2.

magnitude difference in the scattered wavelengths suggests a spatial multisize scattering effect in the 3D graphene sample. These spatial scales can be recognized (see Fig. 1b, c) in the pore structures (tens to hundreds of microns), which act as scattering centers for $\mathrm{THz}$ and far-infrared (FIR) radiation, and branch surface roughness (hundreds of nanometers) which probably plays the major role in the transmittance at infrared and visible wavelengths.

This multisize nature of the scatterers should translate into a strong wavelength dependence of the photon scattering mean free path $\left(l_{s}\right)$. To estimate $l_{s}$, we describe graphene samples as random medium slabs where light propagates between the opposite faces through a combination of scattering and absorption processes. Due to multiple scattering processes, one expects interference effects to be negligible inside the materials, and the semiclassical theory of radiative transfer ${ }^{68-71}$ can be used.

\section{Transmittance of a disordered slab}

The theory of radiative transfer ${ }^{68,69}$ describes a medium where the free propagation of energy is randomly interrupted by scattering processes, changing the propagation direction and causing loss of energy. In this theory the central quantity is the light intensity $I(r, t, \hat{s})$, being the flux per unit solid angle at position $r$ and time $t$, in the direction $\hat{s}$. In a macroscopic isotropic medium such as $3 \mathrm{D}$ graphene samples investigated here, scattering structures such as pores and interconnected layers (see Fig. 1b, c) have equal probability to diffuse light forward or backward the initial propagation direction, as shown by the goniophotometric data presented in $\mathrm{SI}^{65}$. By taking this direction to be along $z$ (see Fig. 4a) and considering the graphene network as a slab between the planes $z=0$ and $z=L$ (see Fig. 4a), the radiative transfer equation for stationary monochromatic light at position $z$ and with a wavevector direction $\hat{s}$, describing the evolution of the intensity due to scattering and absorption, takes the form ${ }^{68}$

$$
l^{\prime} \mu \frac{\partial I}{\partial z}=-I(z, \mu)+\frac{l^{\prime}}{l} \bar{I}(z)
$$

where $l^{\prime}=l_{s} l_{a} /\left(l_{s}+l_{a}\right) \quad\left(l_{s}\right.$ is the mean free path for scattering and $l_{a}$ the absorption length), $\mu \equiv \cos \theta$, with $\theta$ 


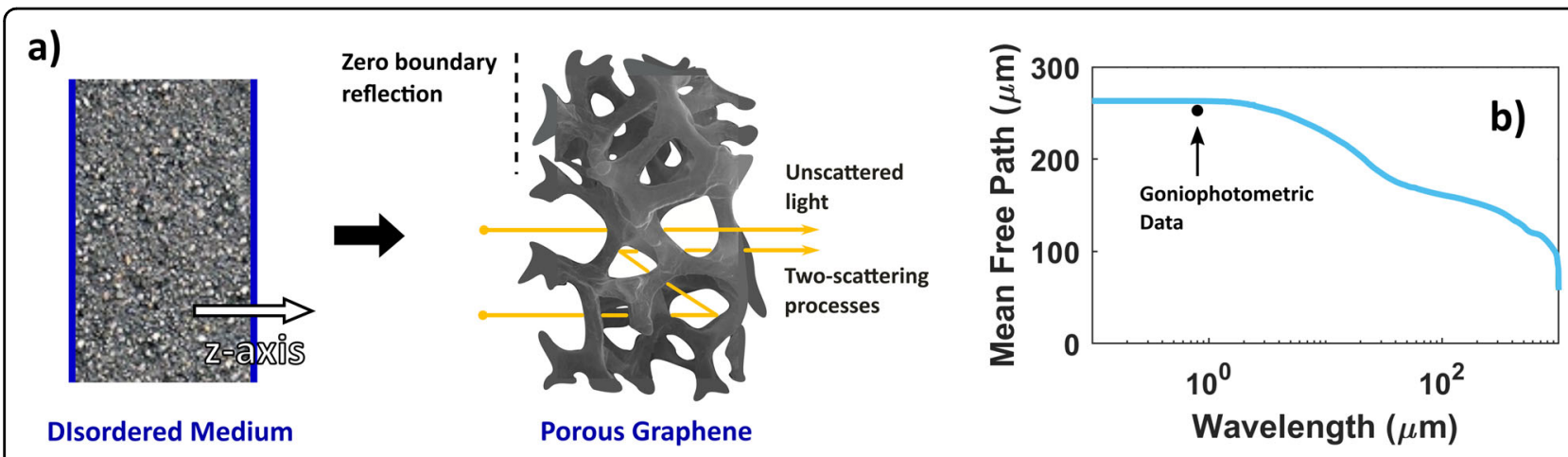

Fig. 4 Radiative transfer theory of a 3D graphene network. a The theory of radiative transfer can be used to describe a disordered medium with reflective boundaries and bulk absorption and scattering processes (left). For a graphene 3D network, the former contribution is zero, while the absorptive processes can be neglected due to propagation in air (right). The remaining scattering contribution can be obtained through an expansion in the number of scattering processes. The first two contributions will take in mind the transmission of non-scattering photons and the two-scattering processes. For the incoherent experimental transmission, the only processes considered are the ones that give raise to photons propagating in the same direction of the incident radiation. $\mathbf{b}$ Mean free path as a function of wavelength for the sample S1, as predicted by the radiative transfer model of Eq. 5 .

being the angle between $\hat{s}$ and the $z$ axis, and $\bar{I}(z)=\frac{1}{2} \int \mathrm{d} \mu I(z, \mu)$. Assuming the reflectance at the slab interfaces $\bar{s}^{1}$ to be zero, the boundary conditions for $\mu>0$ become

$$
I(0, \mu)=I_{0}(\mu), \quad I(L,-\mu)=0
$$

where $I_{0}$ is the incident intensity. As described in $\mathrm{SI}^{65}$, the solutions to this differential problem can be simplified by keeping only the first expansion terms in the multiscattering processes. In particular, the transmittance along the forward direction (the physical quantity we actually measure), $T(L, 1)=I(L, 1) / I_{0}$, of the zero and two-scattering processes is

$$
T(L, 1)=\mathrm{e}^{-L / l_{a}} \mathrm{e}^{-L / l_{s}}\left[1+\frac{L}{2 l_{s}}+\ldots\right]
$$

This equation can be further simplified by assuming that absorption can be neglected. Indeed, due to the similarity of the measured transmittance between undoped S1 and doped S2 samples (see Fig. 2), one expects that most of the light in these samples is diffused. Thus, the $\mathrm{e}^{-L / l_{a}}$ term can be omitted by taking $l_{a} \rightarrow \infty$, i.e., zero absorption. As can be seen in Eq. 5 , the remaining term $L / 2 l_{s} \cdot \mathrm{e}^{-L / l}$ describes the contribution to transmittance coming from the two-scattering paths, which adds to the unscattered photon element $\mathrm{e}^{-L / l_{s}}$ (Fig. 4a). From the measured transmittance of the undoped sample S1 (similar results can be obtained from S2), one can extract the fundamental scattering parameter, i.e., the scattering mean free path $l_{s}$. Its value can be obtained by inverting numerically Eq. 5 up to the third order. The result is shown in Fig. $4 \mathrm{~b}$ where $l_{s}$ is plotted from UV $(200 \mathrm{~nm})$ to
$\mathrm{THz}(1 \mathrm{~cm})$. While $l_{s}$ is nearly flat below $1 \mu \mathrm{m}$, indicating that most of VIS and UV photons are scarcely scattered, it strongly decreases approaching the infrared and terahertz region. Here, scattering processes by the network pores and branches play a major role, inducing a progressive opaqueness in agreement with the transmittance data.

The radiative transfer model was validated by an independent experiment. Indeed, through goniophotometric measurements at a wavelength of $780 \mathrm{~nm}$, extended up to a scattering angle of $170^{\circ}$ and combined with a Monte Carlo analysis (for the complete discussion see $\mathrm{SI}^{65}$ ), we calculate the scattering length at the same wavelength. The result is shown in Fig. $4 \mathrm{~b}$ by a black dot, which is in very good agreement with the estimation (blue curve) based on the radiative transfer equation.

\section{Conclusions}

In this work, for the first time, we have measured the optical properties of technologically important 3D graphene networks, whose outstanding physical and chemical properties find applications in many fields of basic and applied research ${ }^{13-15,54,60-62}$. We have shown how the connectivity and morphology of these materials allow a broadband interaction with light, modifying the transmitted intensity from terahertz to ultraviolet. In particular, the 3D graphene networks behave like a high-pass optical filter due to spatially multiscale random scatterers, corresponding to pores and graphene branches in the 3D network. We have developed a model based on the radiative transfer theory describing the interaction of the network with light, from which we have estimated the photon scattering mean free path. These experimental results prove that $3 \mathrm{D}$ graphene represents a novel disordered photonic material sustaining elastic 
scattering, analogous to some nanofibrils found in biological systems ${ }^{72,73}$.

In this general context, 3D graphene networks open new possibilities to study complex light-matter interaction in random materials. Indeed, analogous to disordered systems appearing in nature ${ }^{72}$, new fabrication processes may produce graphene 3D structures going beyond the Gaussian random scattering paradigm, diffusing light at selected wavelengths or angular distributions, and paving the way for applications in several research and industrial fields.

\section{Methods}

\section{Synthesis of porous graphene and $\mathrm{N}$-doped porous graphene}

The 3D graphene structures were prepared through a porous Ni-based chemical vapor deposition (CVD) growing technique as reported in the work of Ito et $\mathrm{al}^{41,42,47,74}$. Porous Ni was loaded into a quartz tube (external diameter $26 \mathrm{~mm} \times$ internal diameter $22 \mathrm{~mm} \times$ $250 \mathrm{~mm}$ length) as an inner tube which was further inserted into the center of a larger quartz tube (external diameter $30 \mathrm{~mm} \times$ internal diameter $27 \mathrm{~mm} \times 1000 \mathrm{~mm}$ length) in the open-box-type furnace. Ni was annealed at $1000^{\circ} \mathrm{C}$ for 10 min to clean the Ni surface under a mixed atmosphere of H2 (100 sccm) and Ar (200 sccm). Subsequently, graphene/chemically doped graphene was grown at $800^{\circ} \mathrm{C}$ for $10 \mathrm{~min}$ under a mixed atmosphere of $\mathrm{H} 2$ (100 sccm), $\mathrm{Ar}(200 \mathrm{sccm})$, benzene (0.1 mbar, 99.8\%, anhydrous) and/or pyridine (0.2 mbar, $99.8 \%$, anhydrous) as graphene precursors. The furnace was quickly opened and the inner and outer quartz tubes were cooled with a fan down to room temperature. The $\mathrm{Ni}$ substrates were dissolved in a $1.0 \mathrm{M} \mathrm{HCl}$ solution for four days and then etched at $50^{\circ} \mathrm{C}$ in a $1.5 \mathrm{M} \mathrm{HCl}$ solution for 1 day. The resulting graphenes on the solution were repeatedly exchanged with pure water five times and kept on the water for 2 days. Then, they were transferred into 2-propanol for 1 week to dry them with a supercritical CO2 fluid drying method ${ }^{75,76}$. To prevent the collapse and damage of the fragile 3D curved structures, caused by the capillary force of water during the drying process, the graphene samples were immersed in 2-propanol and dried using supercritical $\mathrm{CO}_{2}\left(\mathrm{scCO}_{2}\right)$, in order to substitute 2-propanol with $\mathrm{scCO}_{2}$ without the capillary force taking place. The samples were first transferred to a glass bottle (volume: $5 \mathrm{~mL}$ ) filled with 2-propanol $(400 \mu \mathrm{L})$, which was then placed in an $80 \mathrm{~mL}$ pressure-resistant container (TAIATSU techno Corp). After removing the air inside the container through gaseous $\mathrm{CO}_{2}$ purging, the pressure of the container was gradually increased to $15 \mathrm{MPa}$ by introducing liquid $\mathrm{CO}_{2}$ $\left(5 \mathrm{MPa}, 4{ }^{\circ} \mathrm{C}\right.$, density of $0.964 \mathrm{~g} / \mathrm{mL}$ ) at a flow rate of $20 \mathrm{~mL} / \mathrm{min}(19 \mathrm{~g} / \mathrm{min})$ using a high-pressure plunger pump (NIHON SEIMITSU KAGAKU Co. Ltd, NP-KX540). The $\mathrm{scCO}_{2}$ drying process was carried out at $70{ }^{\circ} \mathrm{C}$, with a constant $\mathrm{CO}_{2}$ flow rate of $5 \mathrm{~mL} / \mathrm{min}(4.8 \mathrm{~g} / \mathrm{min})$, by forming a homogeneous phase of 2-propanol and $\mathrm{scCO}_{2}$ to minimize the capillary force. The pressure was maintained at $15 \mathrm{MPa}$ during the drying procedure for at least $5 \mathrm{~h}$. Once completely dried, the temperature of the system was set at $40{ }^{\circ} \mathrm{C}$ and it was gradually depressurized over $43 \mathrm{~h}$, from $15 \mathrm{MPa}$ down to atmospheric pressure, by gradually venting $\mathrm{CO}_{2}$ from the system.

\section{Characterizations}

In this paper, we measured two nanoporous graphene samples, both with an average pore size of $\approx 100 \mu \mathrm{m}$. The first sample (S1) is undoped, while the second (S2) is doped with nitrogen (see $\mathrm{SI}^{65}$ for Raman characterization). The samples have a thickness of approximately $1 \mathrm{~mm}$ and are composed on average of 12 graphene layers. The 3D structures were characterized by scanning electron microscopy (SEM) and optical microscopy. The SEM images were collected with a MINI-SEM SNE-3200M, operating at an accelerating voltage of $20 \mathrm{kV}$ and with a magnification factor of $\times 150$. Optical images of 3D graphene branches (Fig. 1c), at $\times 150$ magnification, have instead been acquired through a JASCO NR5100 microscope. The graphene resistivities were measured by a standard four-probe method using a semiconductor parameter analyzer (Keysight $\mathrm{B} 1500 \mathrm{~A}$ ) at room temperature. The sample was placed on a $\mathrm{SiO}_{2} / \mathrm{Si}\left(\mathrm{SiO}_{2}: 300 \mathrm{~nm}\right)$ substrate. The electrode was fabricated using $\mathrm{Cu}$ wires and Ag epoxy (H20E, Epoxy Technology). S1 shows a DC conductivity of approximately $0.5 \mathrm{~S} / \mathrm{cm}$, while S2 of approximately $70 \mathrm{~S} / \mathrm{cm}$, which was nearly a factor 140 larger than the undoped value.

Chemical analyses of the graphene samples were conducted by using an X-ray photoelectron spectroscopy (XPS, AXIS ultra DLD, Shimazu) with Al Ka using an $\mathrm{X}$ - ray monochromator (see $\mathrm{SI}^{65}$ ). The samples were pasted on a sample holder without any supports. Raman spectra were recorded by using a micro-Raman spectrometer (Renishaw InVia Reflex 532) with an incident wavelength of $532.5 \mathrm{~nm}$. All samples show a similar Raman spectrum as shown in Fig. S2, S4 whose corresponding frequencies are reported in Table S1. The laser power was set at $0.1 \mathrm{~mW}$ to avoid possible damage or unexpected reduction by laser irradiation.

The transmission of light through the 3D graphene samples was measured using several different spectroscopic setups to span a very broad frequency range, from $\mathrm{THz}$ to UV. Transmittance $T(\omega)$ measurements, from 1 to $70 \mathrm{~cm}^{-1}$, was performed through a $\mathrm{THz}$ timedomain spectrometer (THz-TDS) based on photoconductive (Hamamatsu Photonics) antennas. From 50 to $5000 \mathrm{~cm}^{-1}$, instead, a Vertex $70 \mathrm{v}$ FTIR broadband 
interferometer was used, while the interval from 3000 to $50000 \mathrm{~cm}^{-1}$ has been spanned through a JASCO v-770 IR-UV spectrometer. Data obtained from these measurements were merged to obtain a single broadband transmittance curve, one for each sample (Fig. 2). Moreover, the transmittance of a single polygon of the 3D network was also analyzed through a Vertex70 V FTIR interferometer coupled with an Hyperion3000 IR microscope equipped with a $64 \times 64$ pixel Focal Plane Array MCT detector.

A goniophotometric technique has been used for measuring the light scattered at different angles with respect to the incident light. In this work, we present scattering data at two different wavelengths, $780 \mathrm{~nm}$ and $7 \mu \mathrm{m}$, obtained through a TOPTICA FemtoFiber Pro and a collinear difference frequency generator (DFG) from Light Conversion, respectively. Laser beams of comparable power (few $\mathrm{mW}$ ), scattered across the graphene samples, are collected at various angles after being filtered by a $0.5 \mathrm{~mm}$ radius metallic pinhole at the detection point. The detection setup is based on an NIR photodiode (Thorlabs) and a Gentec pyroelectric, for $780 \mathrm{~nm}$ and $7 \mu \mathrm{m}$ sources, respectively. The distance between the sample and the detector is $30 \mathrm{~cm}$ at every angle. With this geometry, an angle resolution better than $0.1^{\circ}$ is possible. The measurements were thus taken with a minimum scan step of $0.1^{\circ}$. The laser repetition rate and the detected signal are sent to a lock-in amplifier to increase the signal-to-noise ratio and dynamic range of the measurements.

\section{Acknowledgements}

The authors thank Prof. Liangti Qu and Prof. Zhipan Zhang at the Beijing Institute of Technology, China, for the useful discussion about 3D Graphene properties. We thank also Ms. Kazuyo Omura at the Institute for Material Research of Tohoku University, Japan, for XPS measurements. This work has received the financial support of the Bilateral Cooperation Agreement between Italy and China of the Italian Ministry of Foreign Affairs and of the International Cooperation (MAECI) and the National Natural Science Foundation of China (NSFC), in the framework of the project of major relevance 3-Dimensional Graphene: Applications in Catalysis, Photoacoustics and Plasmonics. This work has also been sponsored by JSPS Grant-in-Aid for Scientific Research on Innovative Areas "Discrete Geometric Analysis for Materials Design" (Grant Numbers JP18H04477, JP2OH04628), JSPS KAKENHI Grant Number JP19K22226 and a cooperative program (Proposal No. 202011CRKEQ-0001) of CRDAMIMR, Tohoku University.

\section{Author details}

'Department of Physics, Sapienza University, Piazzale Aldo Moro 5, 00185 Rome, Italy ${ }^{2}$ INFN section of Rome, P.Le Aldo Moro, 2, 00185 Rome, Italy. ${ }^{3}$ INFN - Laboratori Nazionali di Frascati, via Enrico Fermi 54, 00044 Frascati (Rome), Italy. ${ }^{4}$ SBAI Department, Sapienza University, Via Scarpa 16, 00161 Rome, Italy. ${ }^{5}$ School of Materials Science and Engineering, and Institute of Materials Genome \& Big Data, Harbin Institute of Technology, 518055 Shenzhen, P. R. China. Institute of Applied Physics, Graduate School of Pure and Applied Science, University of Tsukuba, Tsukuba, Ibaraki 305-8573, Japan

\section{Competing interests}

The authors declare no competing interests.

\section{Publisher's note}

Springer Nature remains neutral with regard to jurisdictional claims in published maps and institutional affiliations.

Supplementary information The online version contains supplementary material available at https://doi.org/10.1038/s41427-021-00341-9.

Received: 25 March 2021 Revised: 15 September 2021 Accepted: 5 October 2021.

Published online: 19 November 2021

\section{References}

1. Geim, A. K. \& Novoselov, K. S. The rise of graphene. Nat. Mater. 6, 183-191 (2007).

2. Novoselov, K. S. et al. Two-dimensional gas of massless Dirac fermions in graphene. Nature 438, 197-200 (2005).

3. Castro Neto, A. H., Guinea, F., Peres, N. M. R., Novoselov, K. S. \& Geim, A. K. The electronic properties of graphene. Rev. Mod. Phys. 81, 109 (2009).

4. Avouris, P., Chen, Z. \& Perebeinos, V. Carbon-based electronics. Nat. Nanotechnol. 2, 605-615 (2007).

5. Novoselov, K. S. et al. Electric field effect in atomically thin carbon films. Science 306, 666-669 (2004).

6. Balandin, A. A. Thermal properties of graphene and nanostructured carbon materials. Nat. Mater. 10, 569-581 (2011).

7. Loh, K. P., Bao, Q., Eda, G. \& Chhowalla, M. Graphene oxide as a chemically tunable platform for optical applications. Nat. Chem. 2, 1015-1024 (2010).

8. Dreyer, D. R., Park, S., Bielawski, C. W. \& Ruoff, R. S. The chemistry of graphene oxide. Chem. Soc. Rev. 39, 228-240 (2010).

9. Baker, S. N. \& Baker, G. A. Luminescent carbon nanodots: emergent nanolights. Angew. Chem. Int. Ed. 49, 6726-6744 (2010).

10. Xiang, Q., Yu, J. \& Jaroniec, M. Graphene-based semiconductor photocatalysts. Chem. Soc. Rev. 41, 782-796 (2012).

11. Eda, G. \& Chhowalla, M. Chemically derived graphene oxide: towards largearea thin-film electronics and optoelectronics. Adv. Mater. 22, 2392-2415 (2010).

12. Avouris, P. Graphene Electronic and photonic properties and devices. Nano Lett. 10, 4285-4294 (2010).

13. Ito, Y. et al. Three-dimensional porous graphene net-works expand graphenebased electronic device applications. Phys. Chem. Chem. Phys. 20, 6024-6033 (2018).

14. Rezvani, S. J. et al. Structural anisotropy in three dimensional macroporous graphene: a polarized XANES investigation. Diamond Relat. Mater 111, 108171 (2021).

15. Yang, $\mathrm{H}$. et al. Retarding Ostwald ripening to directly cast 3D porous graphene oxide bulks at open ambient conditions. ACS Nano 14, 6249-6257 (2020).

16. Kim, T., Jung, G., Yoo, S., Suh, K. S. \& Ruoff, R. S. Activated graphene-based carbons as supercapacitor electrodes with macro- and mesopores. ACS Nano 7, 6899-6905 (2013).

17. Yang, X., Cheng, C., Wang, Y., Qiu, L. \& Li, D. Liquid- mediated dense integration of graphene materials for compact capacitive energy storage. Science 341, 534-537 (2013).

18. Lin, J. et al. Tour 3-dimensional graphene carbon nanotube carpet-based microsupercapacitors with high electrochemical performance. Nano Lett. 13, 72-78 (2013).

19. Yan, J. et al. Advanced asymmetric supercapacitors based on Ni(OH)2/graphene and porous graphene electrodes with high energy density. Adv. Funct. Mater. 22, 2632-2641 (2012).

20. $\mathrm{Yu}, \mathrm{M}$. et al. Building three-dimensional graphene frameworks for energy storage and catalysis. Adv. Funct. Mater. 25, 324-330 (2014)

21. Fan, Z. et al. Easy synthesis of porous graphene nanosheets and their use in supercapacitors. Carbon 50, 1699-1703 (2012).

22. Chmiola, J., Largeot, C., Taberne, P. L., Simon, P. \& Gogotsi, Y. Monolithic carbide-derived carbon films for micro-supercapacitors. Science $\mathbf{3 2 8}, 480-483$ (2010).

23. Li, Y., Li, Z. \& Shen, P. K. Simultaneous formation of ultrahigh surface area and three-dimensional hierarchical porous graphene-like networks for fast and highly stable supercapacitors. Adv. Mater. 25, 2474-2480 (2013). 
24. $\mathrm{Yu}, \mathrm{G}$. et al. Solution-processed graphene/ $\mathrm{MnO}_{2}$ nanostructured textiles for high-performance electrochemical capacitors. Nano Lett. 11, 2905-2911 (2011).

25. Chen, K., Chen, L., Chen, Y., Bai, H. \& Li, L. Three-dimensional porous graphenebased composite materials: electrochemical synthesis and application. J. Mater. Chem. 22, 20968-20976 (2012)

26. Stoller, M. D., Park, S., Zhu, Y., An, J. \& Ruoff, R. S. Graphene-based ultracapacitors. Nano Lett. 8, 3498-3502 (2008).

27. Kashani, $\mathrm{H}$. et al. Bicontinuous nanotubular graphene- polypyrrole hybrid for high performance flexible supercapacitors. Nano Energy 19, 391-400 (2016).

28. Ji, Kemeng. et al. Lithium intercalation into bilayer graphene. Nat. Commun. 10, 275 (2019).

29. Qiu, Hua-Jun. et al. Metal and nonmetal codoped 3D nanoporous graphene for efficient bifunctional electro- catalysis and rechargeable Zn-Air batteries. Adv. Mater. 31, 1900843 (2019).

30. Sun, B., Huang, X., Chen, S., Munroe, P. \& Wang, G. Porous graphene nanoarchitectures: an efficient catalyst for low charge-overpotential, long life, and high capacity lithium-oxygen batteries. Nano Lett. 14, 3145-3152 (2014).

31. Mukherjee, R. et al. Defect-induced plating of lithium metal within porous graphene networks. Nat. Commun. 5, 3710 (2014).

32. Xiao, J. et al. Hierarchically porous graphene as a lithium-air battery electrode. Nano Lett. 11, 5071-5078 (2011).

33. Guo, $X$. et al. 3D nanoporous nitrogen-doped graphene with encapsulated $\mathrm{RuO}_{2}$ nanoparticles for Li-O2 batteries. Adv. Mater. 27, 6137-6143 (2015).

34. Han, J. et al. Effect of chemical doping on cathodic performance of bicontinuous nanoporous graphene for $\mathrm{Li}^{-} \mathrm{O}_{2}$ batteries. Adv. Energy Mater. 6 , 1501870 (2016).

35. Chen, W., Li, S., Chen, C. \& Yan, L. Self-assembly and embedding of nanoparticles by in situ reduced graphene for preparation of a 3D graphene/ nanoparticle aerogel. Adv. Mater. 23, 5679-5683 (2011).

36. Qin, J. et al. Graphene networks anchored with Sn@graphene as lithium ion battery anode. ACS Nano 8, 1728-1738 (2014).

37. Wei, W. et al. $3 \mathrm{D}$ graphene foams cross-linked with pre-encapsulated $\mathrm{Fe}_{3} \mathrm{O}_{4}$ nanospheres for enhanced lithium storage. Adv. Mater. 25, 2909-2914 (2013).

38. Gong, Y. et al. Graphene-network-backboned architectures for highperformance lithium storage. Adv. Mater. 25, 3979-3984 (2013).

39. Luo, J. et al. Three-dimensional graphene foam supported $\mathrm{Fe}_{3} \mathrm{O}_{4}$ lithium battery anodes with long cycle life and high rate capability. Nano Lett. 13, 6136-6143 (2013).

40. Han, J. et al. Ultrasensitive iron-triggered nanosized FeCoOOH integrated with graphene for highly efficient oxygen evolution. Adv. Energy Mater. 7, 1601933 (2017).

41. Ito, Y., Weitao, C., Fujita, T., Tang, Z. \& Chen, M. High catalytic activity of nitrogen and sulfur co-doped nanoporous graphene in the hydrogen evolution reaction. Angew. Chem. Int. Ed. 54, 2131-2136 (2015).

42. Ito, Y. et al. Correlation between chemical dopants and topological defects in catalytically active nanoporous graphene. Adv. Mater. 28, 1064410651 (2016).

43. Qiu, H. J. et al. Nanoporous graphene with single-atom nickel dopants: an efficient and stable catalyst for electrochemical hydrogen production. Angew. Chem. Int. Ed. 54, 14031-14035 (2015).

44. Lin, Z., Waller, G. H., Liu, Y., Liu, M. \& Wong, C.-P. 3D Nitrogen-doped graphene prepared by pyrolysis of graphene oxide with polypyrrole for electrocatalysis of oxygen reduction reaction. Nano Energy 2, 241-248 (2013).

45. Lin, Z., Waller, G. H., Liu, Y., Liu, M. \& Wong, C.P. Simple preparation of nanoporous few-layer nitrogen-doped graphene for use as an efficient electrocatalyst for oxygen reduction and oxygen evolution reactions. Carbon $\mathbf{5 3}$, 130-136 (2013).

46. Zhang, J., Zhao, Z., Xia, Z. \& Dai, L. A metal-free bi-functional electrocatalyst for oxygen reduction and oxygen evolution reactions. Nat. Nanotechnol. 10, 444-452 (2015).

47. Ito, $\mathrm{Y}$. et al. Bicontinuous nanoporous n-doped graphene for the oxygen reduction reaction. Adv. Mater. 26, 4145-4150 (2014).

48. Xiao, X. et al. Lithographically defined three-dimensional graphene structures. ACS Nano 6, 3573-3579 (2012).
49. Su, Y. et al. Low-temperature synthesis of nitrogen/sulfur co-doped threedimensional graphene frameworks as efficient metal-free electrocatalyst for oxygen reduction reaction. Carbon 62, 296-301 (2013).

50. $\mathrm{Hu}, \mathrm{C}$. et al. Newly-designed complex ternary Pt/PdCu nanoboxes anchored on three-dimensional graphene framework for highly efficient ethanol oxidation. Adv. Mater. 24, 5493-5498 (2012).

51. Yong, Y. C., Dong, X. C., Chan-Park, M. B., Song, H. \& Chen, P. Macroporous and monolithic anode based on polyaniline hybridized three-dimensional graphene for high-performance microbial fuel cells. ACS Nano 6, 2394-2400 (2012).

52. Niu, Z. et al. A universal strategy to prepare functional porous graphene hybrid architectures. Adv. Mater. 26, 3681-3687 (2014).

53. Ito, Y. et al. 3D bicontinuous nanoporous reduced graphene oxide for highly sensitive photodetectors. Adv. Funct. Mater. 26, 1271-1277 (2016).

54. Ito, Y. et al. Multi-functional porous graphene for high-efficiency steam generation by heat localization. Adv. Mater. 27, 43024307 (2015).

55. Harrison, B. S. \& Atala, A. Carbon nanotube applications for tissue engineering. Biomaterials 28, 344-353 (2007).

56. Chen, H., Müller, M. B., Gilmore, K. J., Wallace, G. G. \& Li, D. Mechanically strong, electrically conductive, and biocompatible graphene paper. Adv. Mater. 20, 3557-3561 (2008)

57. Hou, C., Duan, Y., Zhang, Q., Wang, H. \& Li, Y. Bio-applicable and electroactive near-infrared laser-triggered self-healing hydrogels based on graphene networks. J. Mater. Chem. 22, 14991-14996 (2012).

58. Tanabe, $Y$. et al. Electric properties of Dirac fermions captured into 3D nanoporous graphene networks. Adv. Mater. 28, 10304-10310 (2016).

59. Tanabe, $Y$. et al. Dirac Fermion kinetics in three-dimensionally curved graphene. Adv. Mater. 32, 2005838 (2020).

60. D'Apuzzo, F. et al. Terahertz and mid-infrared plasmons in three-dimensional nanoporous graphene. Nat. Commun. 8, 14885 (2017).

61. Giorgianni, F. et al. High-efficiency and low distortion photoacoustic effect in 3D graphene sponge. Adv. Funct. Mater. 28, 1702652 (2018).

62. De Nicola, F. et al. Graphene plasmonic fractal metamaterials for broadband photodetectors. Sci. Rep. 10, 6882 (2020).

63. Zengling, L. et al. Custom-built graphene acoustic-absorbing aerogel for audio signal recognition. ACS Mater. Interfaces 8, 2100227 (2021).

64. Ron, R., Haleva, E. \& Salomon, A. Nanoporous metallic networks: fabrication, optical properties, and applications. Adv. Mater. 30, 1706755 (2018).

65. Tomarchio, L. et al. Supplementary Information of the article: Disordered Photonics Behavior from Terahertz to Ultraviolet of a 3-dimensional Graphene Network. (https:/doi.org/10.1038/s41427-021-00341-9)

66. Mak, K. F., Ju, L., Wang, F. \& Heinz, T. F. Optical spectroscopy of graphene: From the far infrared to the ultraviolet. Solid State Commun. 152, 1341-1349 (2012).

67. Mogera, U. \& Kulkarni, G. U. Twisted multilayer graphene exhibiting strong absorption bands induced by van Hove Singularities. Bull. Mater. Sci. 41, 130 (2018).

68. Chandrasekhar, S. Radiative Transfer (Dover,1960).

69. Ishimaru, A. Wave Propagation and Scattering in Random Media (Academic Press, 1978).

70. Nguyen, V. D., Faber, D. J., van der Pol, E., van Leeuwen, T. G. \& Kalkman, J. Dependent and multiple scattering in transmission and backscattering optical coherence tomography. Opt. Express 21, 29145 (2013).

71. Fujii, $H$. et al. Photon transport model for dense poly-disperse colloidal suspensions using the radiative transfer equation combined with the dependent scattering theory. Opt. Express 28, 22962 (2020).

72. Wiersma, D. S. Disordered Photonics. Nat. Photonics 7, 188-196 (2013).

73. Lee, S. H., Han, S. M. \& Han, S. E. Anisotropic diffusion in Cyphochilus white beetle scales. APL Photonics 5, 056103 (2020).

74. Ito, Y. et al. High-quality three-dimensional nanoporous graphene. Angew. Chem. Int. Ed. 53, 4822-4826 (2014).

75. Adschiri, T., Hakuta, Y. \& Arai, K. Hydrothermal synthesis of metal oxide fine particles at supercritical conditions. Ind. Eng. Chem. Res. 39, 4901-4907 (2000).

76. Sue, $\mathrm{K}$. et al. Synthesis of $\mathrm{Ni}$ nanoparticles by reduction of $\mathrm{NiO}$ prepared with a flow-through supercritical water method. Chem. Lett. 35, 960-961 (2006). 